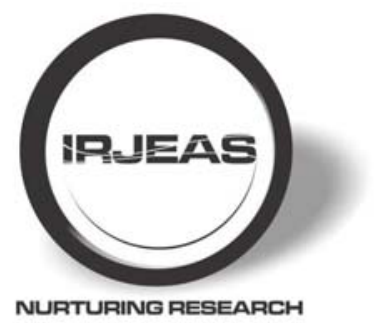

\title{
A WAVELET TRANSFORM AND DATA MINING TECHNIQUE BASED PROTECTION TECHNIQUE FOR MICROGRID
}

\author{
Mamta Narware ${ }^{1}$, Laxman Solankee ${ }^{2}$ \\ ${ }^{* 1}$ M.Tech Scholar, ${ }^{2}$ Asst. Professor \\ Department Of Electrical \& Electronics Engineering, Technocrats Institute of Technology, Bhopal, India
}

*Corr. Author - pmamta24@gmail.com

\begin{abstract}
With the emphasis on reducing carbon footprint and promoting the utilization of non renewable resources of energy in production of electricity, the micro grids have emerged as popular arrangement. Micro grids have empowered the users with flexibility in generating $\&$ controling electricity as per their requirements and have reduced their dependence on utility grid. Also they can now transfer surplus electrical energy to utility grid and can earn monetary gains. Photovoltaic systems have emerged as indispensable alternative over other distributed energy resources that can be inetrated in microgrids due to their simple design, easy to install and easy to maintain features. However the deep penetration of microgrid systems is posing threat to reliable \& optimal operation of main utility grids. It is important to detect the faults on primitive stage $\&$ resove it before it results into catastrophic failure of whole system. This paper presents novel scheme based on wavelet transform \& data mining technique for early detection \& classification of faults that may occur in microgrid. Once the fault is detected \& classified an appropriate protection scheme can be employed to resolve the faults at early stage and thereby improving the overall safe operation of the complete system.
\end{abstract}

Keywords-Microgrid, Detection and Identification, Classification, Fault Diagnosis, Protection System, PV Systems, Wavelet Transform.

\section{INTRODUCTION}

$\mathrm{W}$ ith ever increasing demand for electricity the main transmission networks are now operating near to their stability limits. In an order to address the future demads it is needed to upgrade the exsisting infrastructure which is not an economically viable solution. This has forced to adopt new strategies which includes te generation of electrical energy at the point of consumption it self.

This electrical energy can be generated by utilizing various vastly available renewable energy resources such as wind, sunlight etc. It has led to the concept of Microgrids which is usually consisted of distributed energy resources such as wind mills, fuels cells, PV arrays etc. and dedicated load connected within well defined electrical boundaries. Microgrids have ability of operating while connected to main utility grid \& in isolation also known as islanding mode. Microgrids are offering numerous advantages to consumers $\&$ grid operators in form of less dependence on main grid, flexibility in operation and control, transfer of surplus power to the main grid, improvement in service quality, enahancement in reliability, reduction in pollution \& dependence on conventional energy resources. However the interconnection of microgrid to main utility grid is posing challages in operation \& protection of later. The protection system to be employed must be able to respond to faults of main grid as well as the microgrid.

These faults needed to be detected and classified quickly as possible so that appropoate protection strategy could be pressed into action which can isolate the faulty section \& resolve the fault swiftly.

This paper proposes efficient protection scheme for microgrid based on autocorrelation of three phase current envelopes. It evaluated the envelope of current signal using a squaring and low-pass filtering approach. Then, fault signatures in the microgrid are detected by extracting the information from the distorted envelope by using variance of the autocorrelation function.

Also, the direction of fault is determined using the reactive power. The discrete wavelet transform (DWT) is used to extract the information from the instantaneous voltage and current signal and the standard deviation is calculated from the estimated approximiate coefficients. This information is further used to train the decision tree which is a data mining based model to classify the fault occurred.

The DWT requires lesser computation time for extraction of information from voltage current signal and offers rentention of useful features. The said protection scheme is easy to implement and have shown good performance under looped and radial configuration for both modes of operation i.e. gridconnected and islanded modes. The simulation results obtained testifies that the proposed scheme not only detect, locate, classify, and isolate various types of short-circuit 
faults effectively but also provide backup protection in case of primary protection failure.

\section{LITERATUE REVIEW}

This section elaborates the comparative study of various techniques used in past in the field of fault detection \& classification for microgrid.

James J. Q. Yu et at. [1] proposed the intrelligent fault detection scheme based on wavelet transform and deep neural network. The scehme is intended to provide the information regarding type of fault, phase on which fault has occurred and the location of fault. These information are important for effective protection of microgrid \& the swift recovery from the faulty condition. The wavelet transform extracts the information from branch currents sampled by protective relays then this information is given as input to the deep neural network to develop the information regarding fault. The detailed assessment is carried out on the CERTS microgrid and IEEE 34-bus system for performance eavaluation of the porposed scheme. The results onbatined from simulation reveals its effectiveness with reference to detection, time required for computation $\&$ robustness against uncertainty in measurement. Also when obtained results were compared with preceding research work on the topic, the proposed scheme found better in fault detection \& its location and accuracy for classification of fault. Debi Prasad Mishra et al. [2] used the effective information such as change in energy, entrophy and standard deviation extracted using wavelet coefficients from the current signal at the relaying point. The features are extracted for faulted \& unfaulted conditions for each phase then this data is used to train the decision tree which is conformed for unseen data set for detection of faults in the microgrid. Then wavelet based featured derived from sequence components in addition to features derived earlier from current signals are utilized in building the decision tree for fault classification. Both the Decision Treess are comprehensively assessed on a huge data set of 3860 samples. The results obtained indicates the efficiency and effectiveness of the proposed protection scheme against faults occurring under wide range of operating conditions in microgrid. Shazia Baloch et al. [3] proposed the protection scheme based on autocorrelation of three phase current enevelope of current signal. The hidden information is extracted from distorted enevelop using variance of autocorrelation function to detect the fault signatures in microgrid. MATLAB/Simulink (Version: R2017b) was used to simulate the medium voltage microgrid and study the performance of proposed scheme. The simulation results indicates that the proposed scheme not only identifies the fault, its type but also provides the backup against occurrence of indispensable insurance violation. Sisitha Senarathna et al.[4] discusses the state of the art of various adaptive methods employed in protection of micro grid. They systematically focuses on the wide range of applicability variants, their strengths \& drawbacks.In this paper author elaborated the various considerations accumulated by Microgrids inside the last decade that has turned the fundamental resource into viable business. The ease of installation, control and maintenance is one of the principle explanations behind microgrids popularity. A wide variety of Distributed Generation (DG) options that may include wind turbine, other smaller scale turbine, PV systems, fuel cells etc. which can be easily integrated to form microgrid and make it suitable to operate in both grid connected and islanded modes. There are number of challenges needed to be addressed so as to utilize the maximum capacity of microgrids, and insurance is one of such challange. Different arrangements of insurance were devised, based on advancements in insurance strategies. Versatile assurance is one of such emerging methodology for microgrid insurance. This paper presents an elaborated audit on versatile insurance of microgrids which includes a wide scope of materialness variations taking into account of their qualities, and limitations. It also investigates the paramount explores that applies computational insight to implement versatile insurance.These arrangements have initiated the thorough reclassification of insurance arrangements leading to progressively adaptable and solid framework that can be implemented all inclusive. Lai Lei et al.[5] explained that there is a difference in level of fault current between islanded mode and grid connected mode which ultimately degrades the overcurrent protection startegies. This paper proposes a protection scheme dependent on feature cosine and differential plan. At first feature cosine is proposed which uses equation of ellipse and minimum least squares to enumerate the unified performance of voltage and current. Secondly the difference of feature cosine between healthy \& faulty sections of the microgrid is determined by analyzing the direction of fault current and feature cosine for different fault locations in a microgrid. Then differential direction is defined and hence the faulty section is detected based on feature cosine and differential scheme. Lastly diverse time domain imitations including different modes of operation of microgrid, type of faults, identification of faulty section, and noise influence were studied and results obtained verifies the high accuracy of the proposed protection scheme. M. Amin Zamani et al. [6] discussed the key challenges in protection of microgrid while working in both modes of operation. This paper proposed the protection scheme for low voltage microgrid using microprocessor based relays alongwith details of structure \& design of the relay. The major advantages of the proposed scheme is non requirement of communication or compatible protection devices also it is independent of mode of operation and the magnitude of the fault current. PSCAD/EMTDC software package is used for simulation studies of transient time domain.

\section{PROBLEM DEFINITION}

This proposed work is motivated from the challenges offered by large scale integration of DERs and their operation in islanded mode to cater the emergency power demand ensuring the reliability under faulty conditions or disturbances in the grid. The situation can be overcome by using the discrete wavelet transform (DWT) and data-mining technique for easy fault detection and classification that has been developed for the microgrid. The microgrid have significant edge in the distribution systems but at the same time the challenges in integration of renewable and synchronous DERs made the protection tasks inevitably difficult. Renewable DERs when compared to synchronous DERs have dynamic generation output with limited capability for carrying fault current which is upto 2-3 times the rated current, same in case of synchronous DERs is up to even 10 times. This forms a wide distinction in operating conditions between them. These reasons further makes the associated 


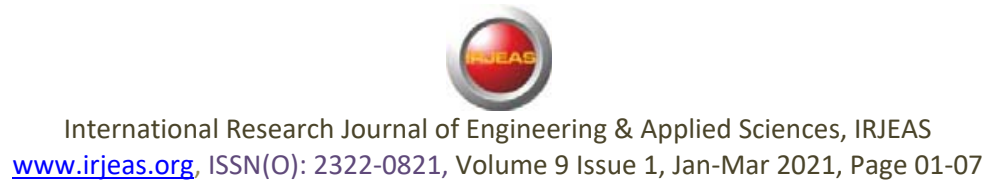

protection issues more complicated. As the fault current magnitude is dependent on mode of operation this leads to vast difference in the level of fault current between islanded and grid-connected mode. Hence, it is needed to formulate accurate and reliable protection strategy which can work effectively under both operating modes and can meet the protection requirements of the microgrid without being affected with the harmonics associated with power electronic interfacing devices and non-linear loads.

\section{PROPOSED METHODOLOGY}

This section elaborates an overview of the proposed methodology which is based on the application of wavelet transofrm and decision tree classfier.

\section{Simulation of Microgrid understudy}

The simulation is carried out for operation of Microgrid performing load leveling and voltage regulation in the local power system. Also the dynamic responses of the Microgrid components are presented when subjected to sudden changes in solar irradiance. Battery energy storage effectivly compensated the fluctuations of PV system output. The proposed model of microgrid also considered the critical compoenents such as solid-state converters for analysis of protection of microgrids, as current rating of most of the converter based DERs are limited to maximum of twice the rated current. This results into limitation on magnitude of fault current to be handeled which inturn creates sensitivity issues in the protection of microgrids.

\section{Genration of fault and other operating Scenario}

The major challenges regarding the protection of microgrids arise from the construction features of a microgrid, which involve large scale presence of converter type DERs and the possibility of two way power flow. That is to be added to the low inertia of the systems especially in the island mode of operation. This makes microgrids easily susceptible to instability due to changes in load and generation.

\section{Development of protection scheme for microgrid}

The enhancement in consistency of the microgrid has been studied by employing auto reclosures in proposed adaptive protection scheme, this enabled the system to recover faster from the faulty condition. The time domain simulations carried out on proposed adaptive protection for its efficiency and effectiveness using MATLAB.

\section{Feature extraction using Wavelet transform}

Wavelet transform has been used for feature extraction in image processing so that the image can be represented in its compacted and inimitable form of matrix vector of single values.

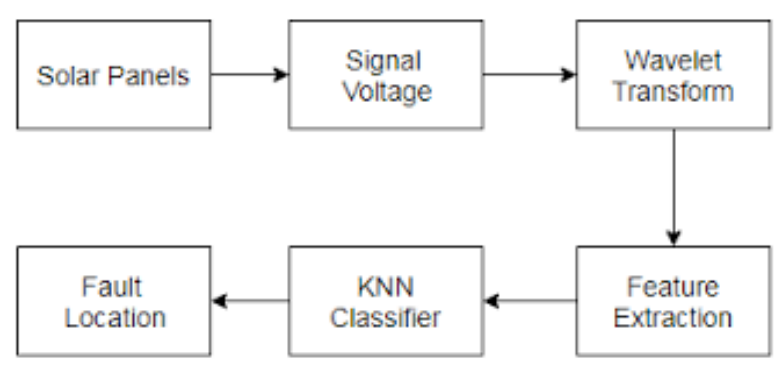

Figure1. Steps involved in Feature extraction using discrete wavelet transform

Fault detection and classification using grid connected and islanded modes using bagged decision tree algorithm

Correlation analysis has been used to select the inout features form the signal processing stage and are used to train the bagged decision trees for detecting the faults in the proposed protection scheme. The technique was tested on a microgrid model.

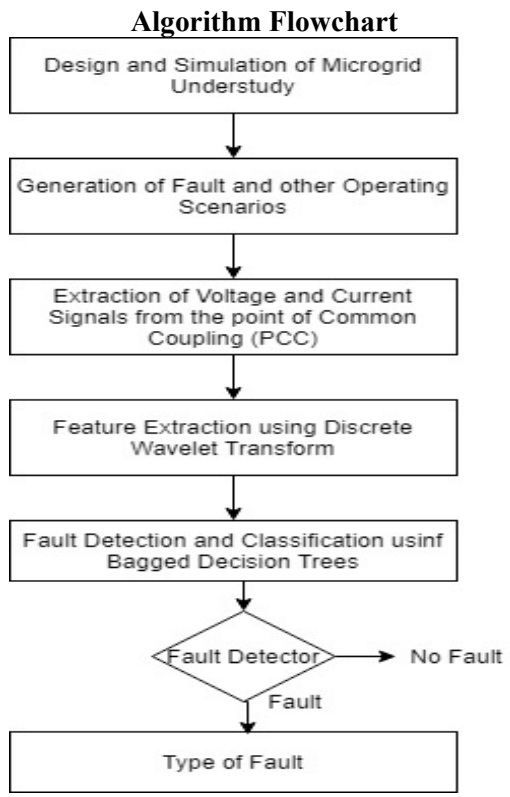

Figure 2. Algorithm flowchart

The proposed algorithm has been shown inabove Figure 2 . The various steps involved in the flowchart are as follows:

Step 1: The work of the proposed algorithm starts from the design and simulation of the microgrid understudy model which is the first step.

Step2: In the second step the function call goes at generation of faults and other operating scenarios.

Step 3: In the third step the control will switch to the extraction of the voltage and current signals from the point of common coupling (PCC) with the help of discrete wavelet transform. 


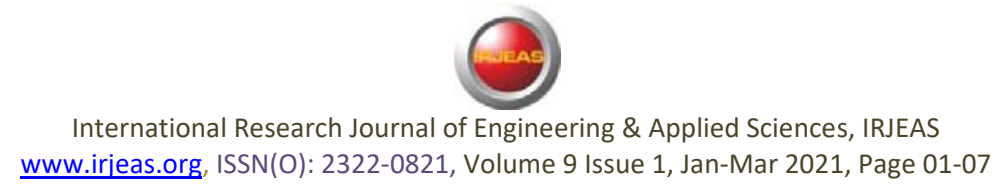

Step 4: In the next step training of bagged decision trees will work thoroughly. The loop will follow the following conditions- if the fault detects then the function control will show the type pf fault which occurs. Otherwise, in second category it will generate output as-no fault.

\section{SIMULATION SETUP}

In this section illustrates the simulation models used in the study:

\section{MATLAB Simulink model of Microgrid under study}

Microgrid may consist of various distributed energy resources (DERs) can operated in islanded mode or grid connected mode. Figure 3 shows the simulink model of the system considered for carrying out investigation. The system shown in figure consists of microgrid connected to the utility grid via point of common coupling (PCC). The Three phase fault has been simulated at bus B3B for both modes of operation of microgrid i.e. grid connected \& island modes. The voltage and current waveform have been recorded for both the case of operations.

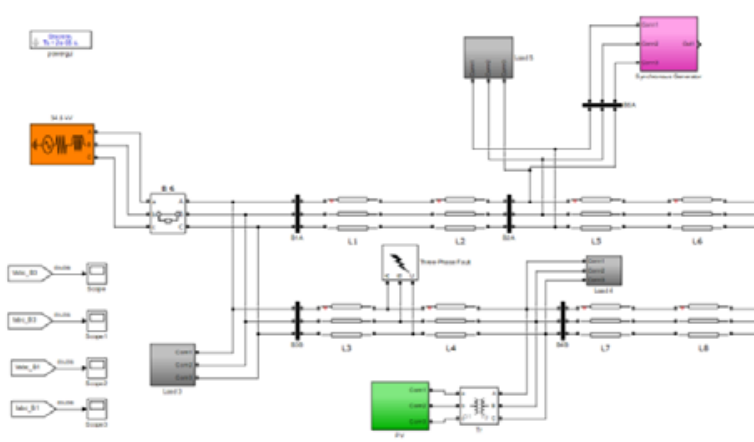

Figure 3. Simulink model of Microgrid considered under study

In the above figure 3 the representation model of the Gridconnecte PV system is shown which is grid-connected photovoltaic system: Mathematical modeling using MATLAB/Simulink.

2. MATLAB Simulink model of PV Array based generator in the microgrid

Figure 4. details the microgrid configuration which consists of PV array, low pass filter, Inverter, Boost Converter Control for Maximum Power Point Tracking, $\mathrm{V}_{\mathrm{sc}}$ Control.

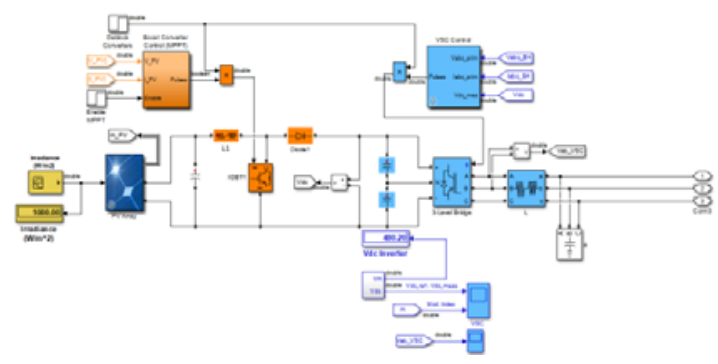

Figure 4. PV array based generator in the Microgrid

\section{I-V and P-V Charateristics of PV Array}

I-V characteristic of PV array represents the relationship between current and voltage for the existing condition of the irradiance. The curve provides the necessary information required to configure the PV system for operating it close to optimal peak power point.

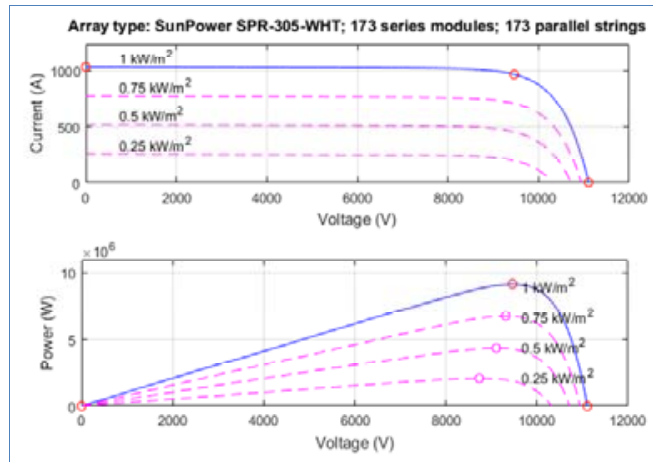

Figure 5. I-V and P-V Characteristics of PV array

Figure 5 shows the I-V and P-V characteristics of the PV array (Sun Power SPR 305-WHT consisting of 173 series modules \& 173 Parallel strings) considered for study. Value of current, voltage, maximum power point (MPP) for different level of irradiance level can be determined using these characteristics.

\begin{tabular}{|c|c|c|c|}
\hline $\begin{array}{c}\text { Irridiance } \\
\text { Level }\end{array}$ & $\begin{array}{c}\text { Power } \\
\text { (W) }\end{array}$ & $\begin{array}{c}\text { Voltage } \\
\text { (V) }\end{array}$ & $\begin{array}{c}\text { Current } \\
\text { (A) }\end{array}$ \\
\hline $1 \mathrm{~kW} / \mathrm{m}^{2}$ & $9 \times 10^{6}$ & 9000 & 1000 \\
\hline $0.75 \mathrm{~kW} / \mathrm{m}^{2}$ & $7 \times 10^{6}$ & 8700 & 750 \\
\hline $0.5 \mathrm{~kW} / \mathrm{m}^{2}$ & $4.5 \times 10^{6}$ & 8600 & 500 \\
\hline $0.25 \mathrm{~kW} / \mathrm{m}^{2}$ & $2 \times 10^{6}$ & 8500 & 250 \\
\hline
\end{tabular}

Table1: Comparative study of power, voltage and current for different levels of irradiance level

Table 1 illustrates the comparative analysis of the Maximum Power Point (MPP) for different levels of irrdiance. The above values have been derived from the I-V and P-V characteristics of the PV array (Sun Power SPR 305-WHT consisting of 173 series modules \& 173 Parallel strings) considered for study.

\section{PARAMETERS USED}

The necessary information from current and voltage signals so obtained due to phase to ground fault occurred while operating in Grid connected mode \& island mode is extracted using wavelet transform. This information is futher used in training of bagged decision tree for the classifying the type of error occurred in the system. 


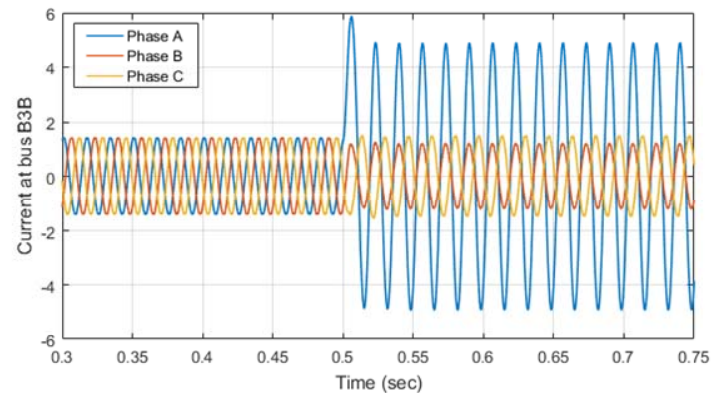

Figure 6. Current at Bus B3B due to A-G fault in the gridconnected mode

The Figure 6 illustrates the current waveform at B3B bus due to phase A to ground (A-G) fault occurred in microgrid system when connected to utility grid. Figure is showing the variation of currents on ordinate while time has been taken on abscissa.

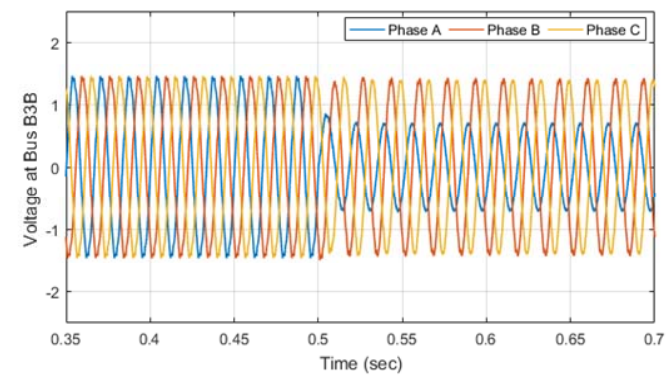

Figure 7. Voltage at Bus B3B due to A-G tault in the gridconnected mode

The Figure 7. is showing the variation of voltage at B3B bus due to phase A to ground (A-G) fault occurred in microgrid system when connected to utility grid. The time has been taken on abscissa while variation of voltage has been taken on ordinate.

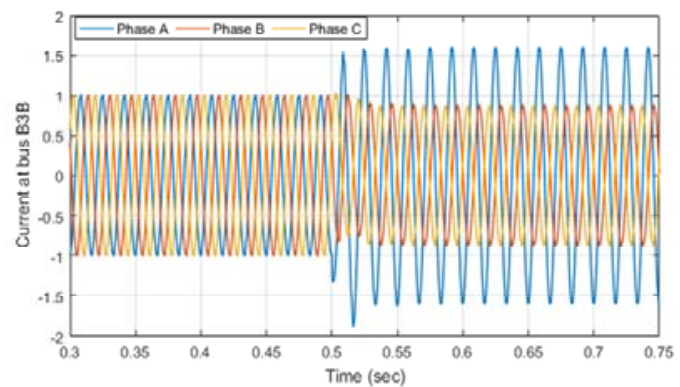

Figure 8. Current at Bus B $3 \mathrm{~B}$ due to A- $\mathrm{U}$ tault in the Utfgrid mode

The Figure 8 shown above illustrates the current waveform at B3B bus due to phase A to ground (A-G) fault occurred in microgrid system when operating in islanded mode. Figure is showing the variation of currents on y axis while time has been taken on $\mathrm{x}$ axis.

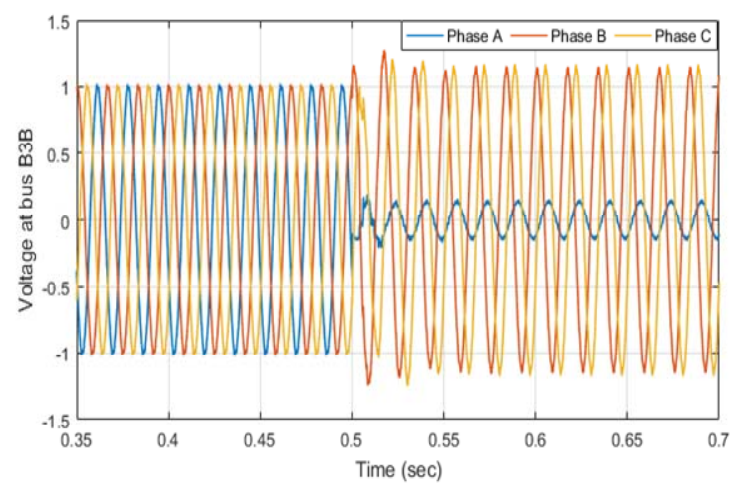

Figure 9. Voltage at Bus B3B due to A-G fault in the Offgrid mode

The Figure 9 shown above illustrates the variation of voltage at B3B bus due to phase A to ground (A-G) fault occurred in microgrid system when operating in islanded mode. Figure is showing the voltage on $\mathrm{y}$ axis while time has been taken on $\mathrm{x}$ axis. The necessary information from current and voltage signals so obtained due to phase to ground fault occurred while operating in Grid connected mode \& island mode is extracted using wavelet transform. This information is futher used in training of bagged decision tree for the classifying the type of error occurred in the system.

In the proposed study, an extensive data set is generated to train and test the data-mining model (using Bagged trees) for developing an accurate and robust classifier to perform the fault detection and classification task. The Baaged decision tree model is trained and tested for different combination of data sets, involving variation in fault parameters and other operating scenarios. For example in combination of (70-30) data set, $70 \%$ of data are considered for training purpose and $30 \%$ of data for testing purpose. The confusion matrix generated for the above system is depicted in Fig. 9, 10 and 11, which provides the comparison results between the actual and predicted faults during testing for the given data set.

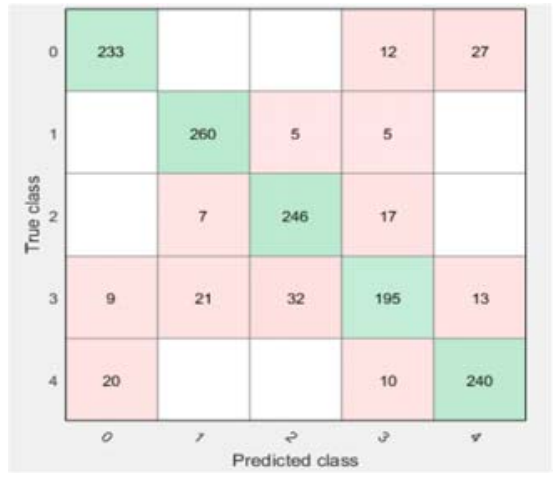

Figure 9: Confusion matrix showing the comparison between true and predicted class 


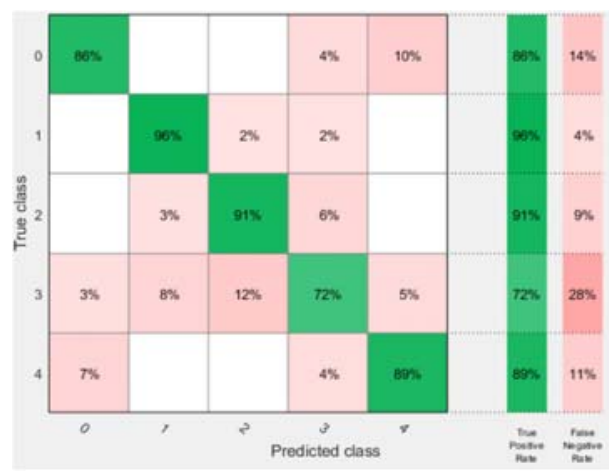

Figure 10: Confusion matrix showing the variation between true positive and true negative rate

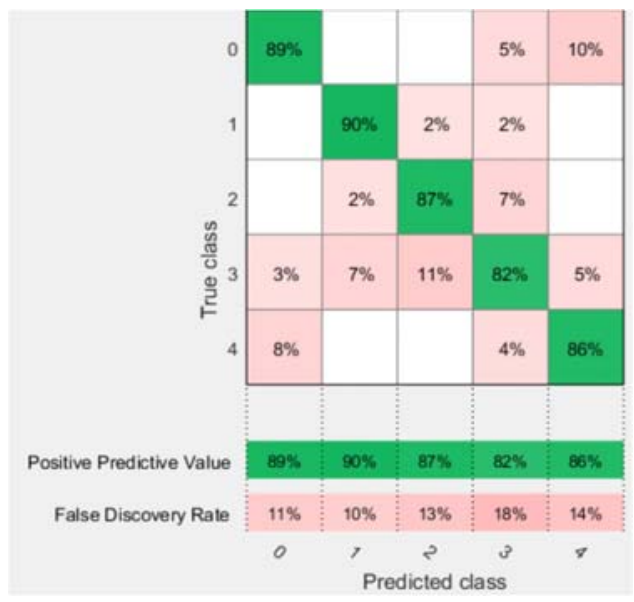

Figure 11: Confusion matrix showing the variation between positive predictive value and false discovery rate

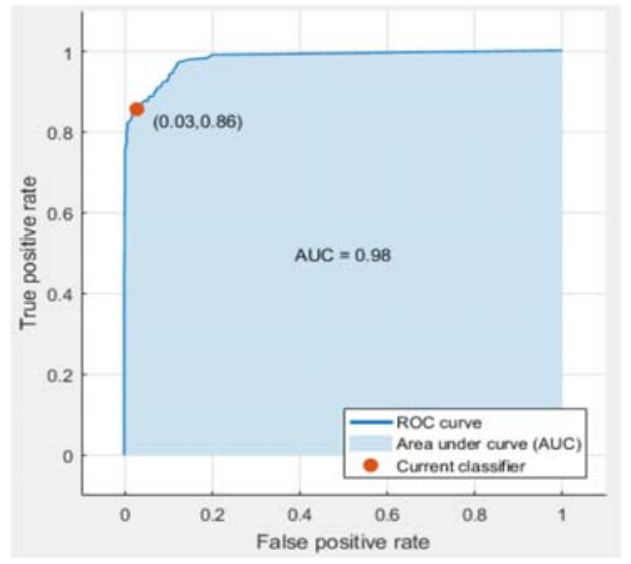

Figure 11: Receiver Operating Characteristic (ROC) curve of Bagged decision tree based classifier
In order to show the robustness of proposed bagged decision tree based classifier in performing the protection task, the Receiver Operating Characteristic (ROC) curve has been depicted in Fig. 11 which shows the ability in performing the intended tasks.

\section{CONCLUSION AND FUTURE WORK}

In this paper PV system based microgrid connected to the utility grid has been considered for study \& simulation. The swift detection \& classification of the faults occurring the microgrid is important in an order to implement the proficient protection startegy for the system.

For the anticipated protection scheme the use of wavelet transform is proposed. It has been used to extract the desired information from the current \& voltage waveforms captured during faults. This information is then utilized in teaching bagged decision trees for identification and classification of faults occurred in the microgrid.

The bagged decision tree are used in decision making process and is one of the important data mining technique. The optimal and safe working of the system can be ensured by adapting suitable protection scheme once the fault is classified accurately.

The proposed scheme of fault identification and classification $\&$ subsequent analysis as elaborated in this paper suggest that devised methodologies are quite capable and practical in approach however some improvements still can be made which are as follows:

1. The microgrid considered for the study \& simulation only consists of the PV arrays. Study can be futher carried out on microgrid constituting of other distributed energy resources such as fuel cell, wind turbines etc.

2. In this dissertation work a phase to groud fault has been simulated for one phase. The results can be verified for other different types of faults such as line to line (L-L), all line to ground (L-L-G) faults.

3. Wavelet transform as a data mining tool uses the regeression as data mining technique. Other data mining techniques such as Tracking patterns, Classification, Association, Clustering etc. may be implemented to extract the information from the data captured during fault in the system.

\section{REFERENCES}

[1].James J. Q. Yu , Member, IEEE, YunheHou, Intelligent Fault Detection Scheme for Microgrids With Wavelet-Based Deep Neural Networks, IEEE TRANSACTIONS ON SMART GRID, VOL. 10, NO. 2, MARCH 2019.

[2].Debi Prasad Mishra, SubhransuRanjanSamantaray, Senior Member, IEEE, and GezaJoos, Fellow, IEEE, A Combined Wavelet and Data-Mining Based Intelligent Protection Scheme for Microgrid, 1949-3053 (C) 2015 IEEE.

[3].Shazia Baloch 1 , Saeed Zaman Jamali 2 , Khawaja Khalid Mehmood 3 , Syed Basit Ali Bukhari 3 , Muhammad Saeed Uz Zaman 1, Arif Hussain 1 and Chul-Hwan Kim 1,*, Microgrid Protection Strategy Based on the Autocorrelation of Current Envelopes Using the Squaring and Low-Pass Filtering Method, 8 May 2020. 
International Research Journal of Engineering \& Applied Sciences, IRJEAS

www.irjeas.org, ISSN(O): 2322-0821, Volume 9 Issue 1, Jan-Mar 2021, Page 01-07

[4].T S SSenarathna and K T M UdayangaHemapala*, Review of adaptive protection methods for microgrids, 11 September 2019.

[5].Lai Lei,1,2 Cong Wang,1 Jie Gao,3 Jinjin Zhao,2 and Xiaowei Wang4,5, , A Protection Method Based on Feature Cosine and Differential Scheme for Microgrid, 10 Mar 2019.

[6].M. A. Zamani, T. S. Sidhu, and A. Yazdani, "A protection strategy and microprocessor-based relay for low-voltage microgrids," IEEE Trans. Power Del., vol. 26, no. 3, pp. 18731883, Jul. 2011.

[7].P. Mahat, C. Zhe, B. Bak-Jensen, and C. L. Bak, "A simple adaptive overcurrent protection of distribution systems with distributed generation," IEEE Trans. Smart Grid, vol. 2, no. 3, pp. 428-437, Sep. 2011.

[8].E. Sortomme, S. S. Venkata, and J. Mitra, "Microgrid protection using communication-assisted digital relays," in Proc. IEEE Power Energy Soc. Gen. Meeting, Minneapolis, MN, USA, 2010, p. 1.

[9].H. Nikkhajoei and R. H. Lasseter, "Microgrid protection," in Proc. IEEE Power Eng. Soc. Gen. Meeting, Tampa, FL, USA, 2007, pp. 1-6.

[10].T. S. Ustun, C. Ozansoy, and A. Zayegh, "Modeling of a centralized microgrid protection system and distributed energy resources according to IEC 61850-7-420," IEEE Trans. Power Del., vol. 27, no. 3, pp. 1560-1567, Aug. 2012.

[11]. T. S. Ustun, C. Ozansoy, and A. Ustun, "Fault current coefficient and time delay assignment for microgrid protection system with central protection unit," IEEE Trans. Power Syst., vol. 28, no. 2, pp. 598-606, May 2013.

[12]. M. A. Zamani, T. S. Sidhu, and A. Yazdani, "A communication-based strategy for protection of microgrids with looped configuration," Elect. Power Syst. Res., vol. 104, pp. 52-61, Nov. 2013.

[13]. S. R. Samantaray, G. Joos, and I. Kamwa, "Differential energy based microgrid protection against fault conditions," in Proc. IEEE PES Innov. Smart Grid Technol. (ISGT), Washington, DC, USA, 2012, pp. 1-7.

[14]. M. A. Haj-ahmed and M. S. Illindala, "The influence of inverter-based DGs and their controllers on distribution network protection," in Proc. IEEE Ind. Appl. Soc. Annu. Meeting, Lake Buena Vista, FL, USA, 2013, pp. 1-9.

[15]. E. Casagrande, W. W. Lee, H. H. Zeineldin, and N. H. Kan'an, "Data mining approach to fault detection for isolated inverterbased microgrids," IET Gener. Transm. Distrib., vol. 7, no. 7, pp. 745-754, Jul. 2013.

[16]. P. Piagi and R. H. Lasseter, "Autonomous control of microgrids," in Proc. IEEE Power Eng. Soc. Gen. Meeting, Montreal, QC, Canada, 2006, p. 8. [17] A. Yazdani and R. Iravani, Voltage-Sourced Converters in Power Systems: Modeling, Control, and Applications. Hoboken, NJ, USA: Wiley, 2010. 